\title{
HIGHLIGHTS
}

RISK FACTORS

\section{Low ghrelin levels in dialysis patients increases mortality risk}

The prevalence of protein-energy wasting (PEW) and anorexia is high among patients with advanced chronic kidney disease. Ghrelin, an orexigenic hormone, contributes to energy regulation, and ghrelin infusion therapy has been shown to improve short-term energy intake and energy balance in malnourished dialysis patients. The results of a new study published in Kidney International shows, for the first time, that low ghrelin levels are associated with a proanorectic phenotype and increased mortality risk in patients undergoing hemodialysis.

\section{Ghrelin is involved not only in eating behavior, but also has beneficial cardiovascular and anti-inflammatory effects... 77}

Ghrelin is primarily produced by endocrine cells lining the stomach, and mediates its effects through the type 1a growth hormone secretagogue receptor. Ghrelin level correlates negatively with an individual's body fat mass and is also affected by nutritional status. This peptide is thought to be the counterpart of the 'satiety hormone' leptin. "Ghrelin is involved not only in eating behavior, but also has beneficial cardiovascular and antiinflammatory effects", notes researcher Peter Stenvinkel.

The investigators of this new crosssectional study measured levels of ghrelin, leptin, PEW (defined as subjective global assessment $>1$ ), and C-reactive protein in 217 patients on hemodialysis. Survival analysis was carried out after a median of 31 months. During this period, 83 deaths occurred, 36 of which were thought to be related to cardiovascular disease. Ghrelin levels were positively associated with adiponectin levels, and negatively associated with age, BMI, and leptin levels, with low levels being more common in men. Patients in the lowest tertile of ghrelin values had a crude hazard ratio of 1.68 for mortality compared with patients with higher ghrelin levels (middle and high tertiles). This risk persisted even after adjustment for age, sex, and dialysis vintage, although it was lost after correction for comorbidities. Regarding all-cause mortality, patients with PEW exhibited a worse outcome in crude and adjusted analyses than patients who were not wasted, with those who also had low ghrelin levels faring the worst. Wasted patients with low ghrelin levels had the highest concentrations of $\mathrm{C}$-reactive protein and leptin, even after correction for BMI; the risk of mortality from cardiovascular disease in this group of patients was 2.63 times higher than that in wasted patients with high levels of ghrelin. This analysis identifies a group of patients who could potentially benefit from ghrelin administration.

The study has a number of limitations, and the findings will need confirmation in larger and incident patient cohorts. However, Stenvinkel comments "if our findings can be confirmed in other patient groups, it may be possible to select patients in whom ghrelin therapy might be particularly useful." The authors suggest that, in the future, it would be interesting to study whether variations in the ghrelin gene have an impact on the uremic phenotype.

Helene Myrvang

Original article Carrero, J. J. et al. Protein-energy wasting modifies the association of ghrelin with inflammation, leptin, and mortality in hemodialysis patients. Kidney Int. doi:10.1038/ki.2010.487 